\title{
Optimization of Electrically Pumped Microdisk Lasers Integrated with a Nanophotonic SOI Waveguide Circuit
}

\author{
J. Van Campenhout ${ }^{1}$, P. Rojo-Romeo ${ }^{2}$, P. Regreny ${ }^{2}$, C. Seassal ${ }^{2}$, D. Van Thourhout ${ }^{1}$, \\ L. Di Cioccio ${ }^{3}$, J.-M. Fedeli ${ }^{3}$ and R. Baets ${ }^{1}$ \\ ${ }^{I}$ Ghent University-IMEC, Photonics Research Group, Sint-Pietersnieuwstraat 41, 9000 Ghent, Belgium \\ ${ }^{2}$ Institut des Nanotechnologies de Lyon, Ecole Centrale de Lyon, 36 Avenue Guy de Collongue, 69134 Ecully, France \\ ${ }^{3}$ CEA-DRT/LETI, 17 Rue des Martyrs, 38054 Grenoble cedex 9 - France. \\ joris.vancampenhout@intec.ugent.be
}

\begin{abstract}
Electrically-injected continuous-wave lasing was achieved in InP-based microdisks coupled to a sub-micron silicon-on-insulator waveguide, with $0.6 \mathrm{~mA}$ threshold current and up to $7 \mu \mathrm{W}$ coupled output power. We present strategies to improve the device performance.

(C)2007 Optical Society of America

OCIS codes: (140.5960) Semiconductor lasers; (250.5300) Photonic integrated circuits
\end{abstract}

\section{Introduction}

In recent years, silicon-on-insulator (SOI) has emerged as a promising platform for passive photonic functions due to the transparency of silicon at telecom wavelengths, its high refractive index contrast and the fact that complementary metal oxide semiconductor (CMOS) technology can be used for fabricating photonic devices with sub-micron features. A major obstacle for large-scale silicon-based electronicphotonic integration is the absence of a compact and efficient silicon-based light source, due to the indirect band gap of silicon. Some strategies for light emission in silicon have been demonstrated. However, compact and efficient active devices don't seem feasible in the short term. Efficient active photonic functionality can be added to the SOI platform by bonding a thin film of direct band gap material such as InP on top of it. Lasers and detectors can be fabricated in this thin film and can be coupled to the SOI waveguide circuit [1]. Some applications however, such as on-chip optical interconnect, can strongly benefit from dense electronic-photonic integration. Hence, the laser footprint and power consumption should be made very small. Therefore, our work focuses on the heterogeneous integration of ultra-compact InP-based microdisk lasers on the silicon platform, using bonding technology. With this approach, CMOS technology can be used to fabricate and integrate thousands of low-power microlasers on a single die. In this paper we report on first experimental results, showing continuous-wave lasing at room temperature, with substantial coupling into the SOI waveguide. We also discuss the possibility to drastically improve device performance.

\section{Device structure and lasing characteristics}

A schematic representation of the laser structure is shown in figure 1a. A microdisk is etched in a thin InPbased layer bonded on top of a SOI waveguide structure. The fundamental whispering gallery modes are confined to the edges of the microdisk. As a result, a top metal contact can be placed in the centre of the microdisk, without introducing extra optical losses. The bottom contact can be placed on a thin lateral contact layer: this layer will cause no substantial additional optical losses, provided it is sufficiently thin. Another issue in the design of electrically injected thin-film microlasers is how to make a p-type contact with low contact resistance. In a classic substrate laser, this is done by using heavily doped, low-bandgap contact layers. This cannot be done for a thin-film laser structure, as this would cause excessive internal absorption losses. Therefore, we implemented a quaternary Q1.2 tunnel junction (TJ) in combination with another n-type contact, instead of low-bandgap p-type contact layer. The laser mode is evanescently coupled to an underlying SOI wire waveguide, aligned with the outer edge of the microdisk. The wire waveguide has a width of $550 \mathrm{~nm}$ and a thickness of $220 \mathrm{~nm}$. The bonding layer thickness is approximately $130 \mathrm{~nm}$. The InP-based epitaxial layer structure is about $1 \mu \mathrm{m}$ thick and contains three compressively strained InAsP 6-nm quantum wells in 20-nm Q1.2 barrier layers.

For microdisk lasers with 7.5 - $\mu \mathrm{m}$ diameter, we observed continuous-wave lasing at room temperature. Figure $1 \mathrm{~b}$ shows the output power and the device voltage versus the input current. The threshold current is $0.6 \mathrm{~mA}$, which is equivalent with $1.35 \mathrm{kA} / \mathrm{cm}^{2}$, under the assumption of uniform injection. The threshold 


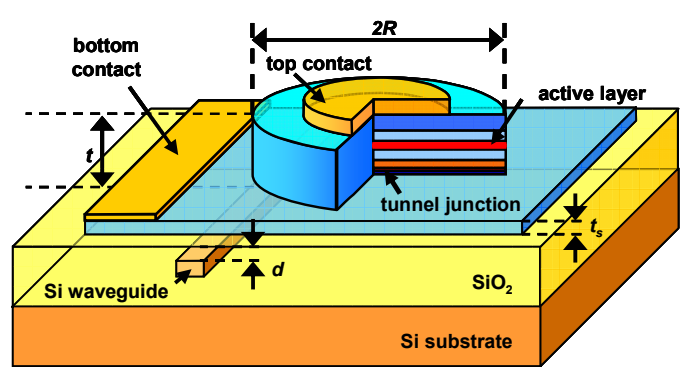

(a)

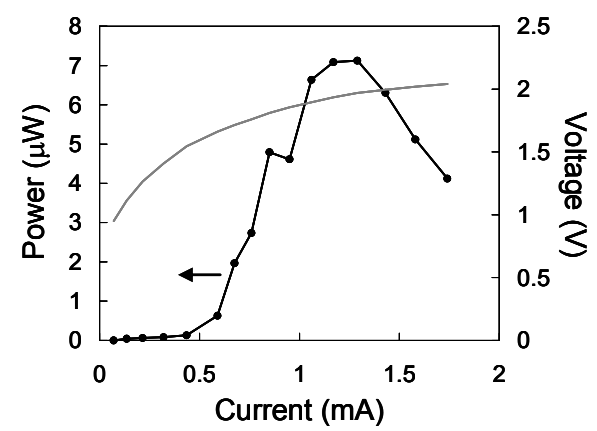

(b)

Fig. 1. Schematic representation of the heterogeneous microdisk laser structure (a) and measured continuous-wave lasing characteristics at room temperature for a $7.5-\mu \mathrm{m}$ device (b).

voltage is about $1.7 \mathrm{~V}$. The output power was collected at one end of the output SOI waveguide, using a fibre grating coupler. The slope efficiency was estimated to be $15 \mu \mathrm{W} / \mathrm{mA}$, and the maximum continuouswave output power is about $7 \mu \mathrm{W}$. In pulsed operation, output peak powers up to $100 \mu \mathrm{W}$ have been measured. The early thermal roll-over is caused by a high thermal resistance, which was found to be $10 \mathrm{~K} / \mathrm{mW}$. The sample contained microdisk lasers with variable top metal contact sizes (5.3-6.3 $\mu \mathrm{m})$. It was found that laser performance depends strongly on the position and size of the top metal. The top contact was misaligned by about $400 \mathrm{~nm}$ during lithography. As a result, only the lasers with smallest top contact showed low-threshold continuous-wave lasing. For bigger top contacts, laser performance was worse due to optical absorption loss at the misaligned top metal.

\section{Device optimization}

Dense integration of these microdisk lasers in an electronic-photonic integrated circuit requires that the power consumption is made sufficiently small. For on-chip applications, we expect that the needed output power will be in the range 1-100 $\mu \mathrm{W}$. We have analyzed the power consumption for such output powers for an optimized microdisk laser design. In this analysis, we neglected self-heating effects. While this effect strongly limits output power for our current devices, we believe that by a incorporating a thermal heat sink structure, self-heating could be strongly suppressed, in particular for optimized lasing structures with output powers below $100 \mu \mathrm{W}$. The input power $P_{i n}$ was calculated for different required output powers $P_{u}$, using the conventional expressions for laser threshold and laser efficiency, and a theoretical assessment of the voltage-current characteristic. For each output power, three design parameters were optimized: the disk radius, the coupling loss into the SOI waveguide, and the doping level in the p-side of the tunnel junction. The bend loss increases exponentially with decreasing disk diameter and therefore poses a limit on the minimum device size. The actual dependence on disk radius was calculated using FDTD and eigenmode expansion techniques. The coupling loss obviously has a large impact on slope efficiency and threshold. For the optimization, it was assumed that arbitrary coupling coefficients can be obtained. This assumption was verified by 3D FDTD simulations, which show that reducing the bonding layer down to $50 \mathrm{~nm}$ yields coupling losses that exceed the optimized values. Finally, the doping level of the tunnel junction has a strong impact on the device voltage, as it is known that the tunnel efficiency strongly increases with increasing doping of the p-side of the tunnel junction. However, internal optical losses also scale with this doping level $P$, due to intervalence band absorption, which is given by $\alpha_{I V B A}=k_{p} P$. The dependence between doping level and TJ resistivity is based on the model in [2]. The important parameters used in the optimization can be found in table 1. The optical confinement factors and internal losses were calculated with a one-dimensional eigenmode expansion tool. Unidirectional output in the SOI waveguide was assumed, which should be possible by incorporating a well positioned mirror in one part of the SOI wire.

The result of the optimization is shown in figure 2. Three cases were considered: one with an additional $10 \mathrm{~cm}^{-1}$ scattering loss, a second without additional scattering loss and a third without scattering loss and with an increased lateral injection efficiency (x2), which might be obtained by etching a hole in the centre of the disk. The optimized disk radii are in the range $2.6-3 \mu \mathrm{m}$, the TJ doping levels are in the range $5-$ $1010^{18} \mathrm{~cm}^{-3}$, while the optimized coupling losses are in the range $3-20 \mathrm{~cm}^{-1}$. For $100 \mu \mathrm{W}$ output power, 
Table. 1. Parameter values used for calculations

\begin{tabular}{|l|l|l|l|l|l|}
\hline Parameter & Value & Parameter & Value & Parameter & Value \\
\hline $\mathrm{A}$ & $10^{8} \mathrm{~s}^{-1}$ & $\mathrm{G}_{0}$ & $1500 \mathrm{~cm}^{-1}$ & $\mathrm{k}_{\mathrm{p}}$ & $4010^{-18} \mathrm{~cm}^{2}$ \\
\hline $\mathrm{B}$ & $210^{-10} \mathrm{~cm}^{-3} \mathrm{~s}^{-1}$ & $\mathrm{n}_{0}$ & $1.510^{18} \mathrm{~cm}^{-3}$ & $\alpha_{0}$ & $7.5 \mathrm{~cm}^{-1}$ \\
\hline $\mathrm{C}$ & $1.610^{-28} \mathrm{~cm}^{-6} \mathrm{~s}^{-1}$ & $\Gamma_{\mathrm{QW}}$ & $4.32 \%$ & $\Gamma_{\mathrm{TJ}}$ & $0.85 \%$ \\
\hline$\eta_{\mathrm{v}}$ & 0.7 & $\eta_{\mathrm{L}}$ & 0.36 & & \\
\hline
\end{tabular}

(a)

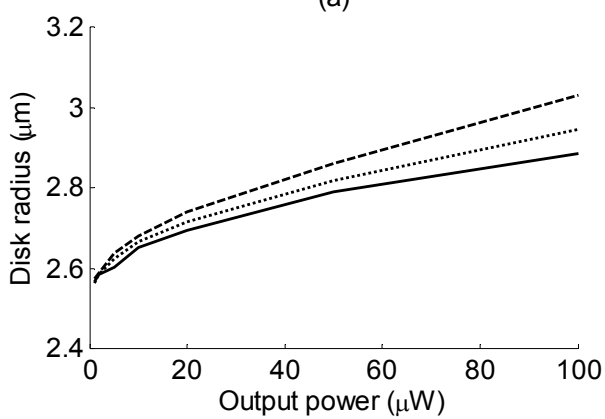

(c)

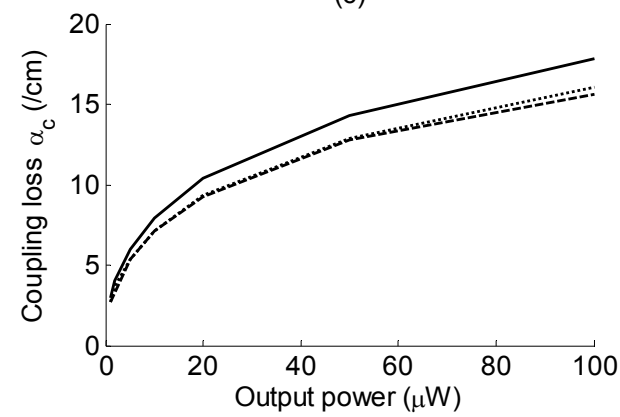

(b)

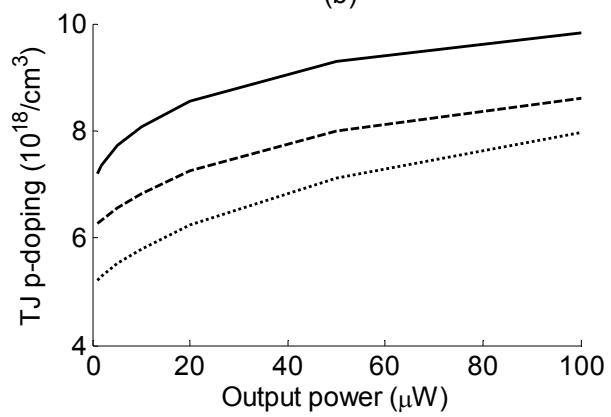

(d)

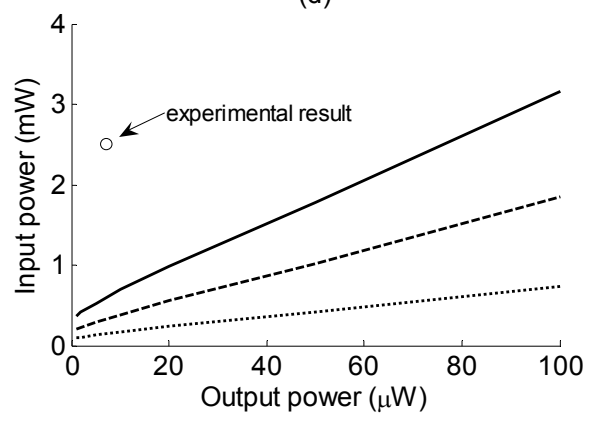

Fig. 2. Optimizing the microdisk radius (a), tunnel junction doping (b) and coupling loss (c) yields devices with $100 \mu \mathrm{W}$ output power for an input power of a few $\mathrm{mW}$. Three different situations were considered: $10 \mathrm{~cm}^{-1}$ additional scattering loss (solid lines), no scattering loss

(dashed lines) and no scattering loss with increased injection efficiency (dotted lines).

the needed input powers would respectively be $3.16,1.85$ and $0.73 \mathrm{~mW}$, enabling the integration of thousands of microlasers on a single chip with a total power consumption of only a few W. This low power consumption also lowers the self-heating effect. A reduction of the thermal resistance by an order of magnitude would eliminate self-heating for all three cases. For comparison, figure 2 also shows the current experimental result.

\section{Conclusions}

We have demonstrated electrically-injected continuous-wave lasing at room temperature in microdisk lasers integrated on and coupled to a nanophotonic silicon-on-insulator waveguide circuit. The microdisks were defined in a thin InP-based film, which was directly bonded to the SOI-wafer. Simulations show that the device performance can be greatly enhanced by further optimizing the coupling efficiency, the tunnel junction doping levels and the lateral injection efficiency. We conclude that this device has great potential for dense and cost-effective electronic-photonic integration.

\section{References}

[1] A.W. Fang, H. Park, O. Cohen, R. Jones, M.J. Paniccia, and J.E. Bowers, "Electrically pumped hybrid AlGaInAs-silicon evanescent laser," Opt. Express 14, 9203-9210 (2006)

[2] Mehta, M. et al. "Electrical design optimization of single-mode tunnel-junction-based long-wavelength VCSELs," IEEE J.

Quantum Electron. 42, 675-682 (2006) 